\title{
Actitudes hacia la conservación del medio ambiente en varones y mujeres de Cañete ${ }^{1}$
}

\author{
Toward environmental conservation in estudents men and women from Cañete
}

\author{
Cindy Fiorella Gutiérrez Jiménez ${ }^{2}$ \\ Universidad Femenina del Sagrado Corazón - Lima, Perú \\ https//orcid.org/0000-0003-0595-8915
}

\begin{abstract}
RESUMEN
El presente estudio compara las actitudes hacia la conservación del medio ambiente en escolares, según sexo y zonas de estudio, es decir urbanas y rurales de la UGEL 08 de la provincia de Cañete; en 743 escolares del nivel secundaria, a los cuales se aplicó la Escala de actitudes hacia la conservación del medio ambiente (Yarlequé, 2004), adaptado para tal fin y, demostrando la validez y confiabilidad de dicha prueba. El diseño del estudio es descriptivo comparativo. Resultados: Se obtiene diferencia en la dimensión cognitiva entre estudiantes de zona urbana y de zona rural a favor de los estudiantes de zona rural, y un mayor compromiso en las actitudes mujeres de zona rural, cuyas características personales son más favorables hacia la conservación del medio ambiente.
\end{abstract}

\section{Palabras clave}

Actitudes, conservación del medio ambiente, Unidad de Gestión Local, escuela, urbano y rural

\section{ABSTRACT}

This study compares school students'attitudes towards environmental conservation, according to their gender and geographical location: urban and rural school from UGEL 08 in the province of Cañete. In this study, 743 secondary school students were administered the Attitudes towards Environmental Conservation Scale (Yarlequé, 2004), which was adapted for this purpose, demonstrating the validity and reliability of this test. The design of the study is descriptive and comparative. Results: There is a difference in the cognitive dimension between urban and rural students, favoring the latter, and a greater commitment among the female rural students, whose personal qualities are more favorable to environmental conservation.

\section{Keywords}

Attitudes, environmental conservation, Local Management Unit, school, urban, rural

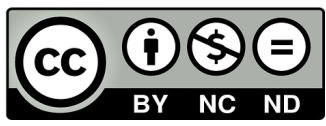

Cualquier uso que se haga de este artículo debe incluir: Autor / Título original de la publicación / ISSN.

\footnotetext{
1. Artículo basado en la tesis de la autora titulada: Actitudes hacia la conservación del medio ambiente en varones y mujeres de Cañete sustentada para obtener el grado académico de Maestra en Psicología con mención en Diagnóstico e Intervención Psicoeducativa, en el año 2019, en la Universidad Femenina del Sagrado Corazón, UNIFÉ, Lima, Perú 2. Magister en Psicología. cindy.gutierrezj@unife.pe
} 


\section{INTRODUCCIÓN}

El presente estudio está centrado en las actitudes hacia la conservación del medio ambiente en estudiantes de la Ciudad de Cañete de las zonas urbanas y rurales. Al respecto hay que tomar en cuenta sobre la importancia del tema toda vez que la ciudad de Lima es una de las ciudades más contaminadas de Sudamérica, señalado por Holguín (2014), sin que ninguna gestión, municipal, regional o gubernamental pueda revertir dicha situación. Siguiendo con Holguín, en la ciudad de Lima se genera más de 7.400 toneladas de basura cada día $\mathrm{y}$ un tercio de esta basura termina en las calles, en la medida que no se recoge.

A esto hay que agregar el gran consumo de agua por los pobladores; según las Organización de las Naciones Unidas (citado por Holguín, 2014) se calcula 50 litros de agua por persona cada día, sin embargo, no llega a ciertas poblaciones; las aguas residuales no son tratadas $\mathrm{y}$ muchas veces son empleadas para el riego de parques y jardines.

Zinmermann (2005) manifiesta que las actuales generaciones han sido educadas bajo la orientación del derroche $\mathrm{y}$ del consumo irracional, así como el aprovechamiento sin control de los recursos naturales. Evidentemente, el cuidado del medio ambiente corresponde a un proceso educativo y psicológico, que se inicia en la familia y en las aulas educativas, en la cual la actitud comportamental se ve fortalecida. La Organización de las Naciones Unidas (ONU, 2005), en su informe sobre el desarrollo del milenio, hace notar que a nivel internacional se ve un gran interés en cuidar y proteger el medio ambiente, habiéndose fijado el objetivo de disminuir ostensiblemente el número de personas sin acceso al agua potable y de esta manera lograr el saneamiento básico, en años próximos. A pesar de la meta enunciada no se aprecia aún el avance que se esperaba.

El Índice de Desarrollo Humano publicado por el Programa de las Naciones Unidas para el Desarrollo (PNUD, 2011) promueve el concepto de sostenibilidad y equidad como un factor importante para un mejor futuro para todos. Hay que anotar que el concepto de sostenibilidad y equidad descansa sobre tres pilares: desarrollo económico, social y ambiental.

En lo que respecta al contexto educativo, el Ministerio de Educación (MINEDU, 2019) resalta la labor de los padres y madres de familia cuando son convocados para el mantenimiento del colegio, estableciéndose un diálogo sostenido entre los mismos padres de familia, los maestros y autoridades del centro educativo. Mediante esta acción de interrelación y comunicación se nota cambios actitudinales en el arreglo y conservación de las instituciones educativas. Observándose el magnífico rol de la mujer, en el mejoramiento del medio ambiente institucional y ambiental, es decir que la actitud de las mujeres es de suma importancia en el cambio de actitudes de los niños.

Estas consideraciones demuestran no solo que el tema sea importante sino fundamentalmente vital, ya que está de por medio la sobrevivencia de la especie humana. Es por ello que la formulación del estudio se orienta a la siguiente pregunta ¿Existe diferencia en las actitudes hacia la conservación del medio ambiente entre estudiantes de escuelas rurales y urbanas de la UGEL 08 del nivel secundario? 
OBJETIVOS DE LA INVESTIGACIÓN

\section{Objetivo general}

Comparar las actitudes hacia la conservación del medio ambiente entre estudiantes de escuelas rurales y urbanas de la UGEL 08 del nivel secundario de la provincia de Cañete.

\section{Objetivos específicos}

- Comparar las actitudes hacia la conservación del medio ambiente en las dimensiones cognitiva, reactiva y afectiva entre los estudiantes del nivel secundario de escuelas rurales y urbanas de la UGEL 08 de la provincia de Cañete.

- Comparar las actitudes hacia la conservación del medio ambiente entre estudiantes varones y mujeres de la zona rural de la UGEL 08 de la provincia de Cañete.

- Comparar las actitudes hacia la conservación del medio ambiente entre estudiantes varones y mujeres de la zona urbana de la UGEL 08 de la provincia de Cañete.

- Comparar las actitudes hacia la conservación del medio ambiente entre estudiantes varones de zona rural y varones de la zona urbana de la UGEL 08 de la provincia de Cañete.

- Comparar las actitudes hacia la conservación del medio ambiente entre estudiantes mujeres de zona rural y mujeres de la zona urbana de la UGEL 08 de la provincia de Cañete.

\section{INVESTIGACIONES SOBRE EL TEMA}

Galli, Bedim, Bolzan de Campos y Castellá Sarriera (2013) estudiaron el comportamiento proambiental de niños de 8 a 12 años del sur de Brasil, según sexo, tipo de escuela, local de residencia y edad. Se aplicó la Escala de Comportamiento Proambiental y se encontró que las "las chicas tienden a desarrollar más conductas favorables al ambiente, así como los niños más pequeños, los que viven en ciudades del interior y los que frecuentan escuelas públicas" (p. 459). Un aspecto importante es observar que la preocupación por el tema ambiental genera, asimismo, participación en actividades proambientales.

Kals (2009) realizó estudios sobre el papel de la afinidad emocional hacia la naturaleza en adolescentes y su relación con la voluntad de proteger el medio ambiente; para lo cual tomó como muestra de estudio a 207 adolescentes de Alemania y Lituana. Demostró que existe una relación positiva entre la afinidad emocional hacia la naturaleza, y la voluntad de proteger el medio ambiente, contribuyendo significativamente a tener un compromiso más claro con el medio ambiente.

En nuestro medio, Edquen e Ybañes (2013) estudiaron la influencia del programa educativo, sobre la disminución de la contaminación de desechos o desperdicio en el aprendizaje teórico práctico en escolares de sexto grado, contando con 122 estudiantes divididos en grupo experimental y de control. Se utilizó como instrumentos, el cuestionario y lista de cotejo. Se logró observar las diferencias estadísticas en el proceso de aprendizaje teórico como práctico entre los grupos estudiados, a favor del grupo experimental, donde se aplicó el programa en mención.

Pereyra (2008) realizó una investigación sobre la relación entre responsabilidad ciudadana y las actitudes hacia el medio 
ambiente en 4,667 personas entre 18 y 65 años de edad, en la ciudad de Lima. Los resultados demuestran que la percepción de diferenciación social no se relaciona con la actitud hacia la conservación del medio ambiente, la generosidad en tanto altruismo prevalece y es el valor más importante para producir cambios en el comportamiento de las personas sobre el cuidado de este medio, y en lo que se refiere al género, se encontró que no guarda relación con la actitud de respeto hacia el medio ambiente y el desarrollo sostenible.

Yarlequé (2004) estudió las actitudes hacia la conservación ambiental que tienen los estudiantes de secundaria. Se diseñó y validó una escala de tipo Likert y se aplicó a 3,837 estudiantes procedentes de ocho departamentos del Perú. Sus puntajes fueron comparados según el lugar de residencia, nivel de instrucción, edad y género Los resultados demuestran la existencia de diferencias actitudinales entre los escolares de las tres regiones naturales del Perú, mostrando los estudiantes de la Costa actitudes más favorables hacia la conservación ambiental que los de la Sierra y Selva, y estos mejores que los de la Sierra. Sin embargo, en lo que respecta al nivel de instrucción y edad, estas variables parecen no tener un rol distintivo en las actitudes hacia la conservación ambiental.

Chapilliquen (2007) aplicó el programa "salvemos la tierra" en la formación y consolidación de actitudes positivas hacia la conciencia ecológica en 76 estudiantes del I.E No 1057 del distrito de Lince. Los resultados muestran un efecto positivo en formar y consolidar actitudes positivas hacia la conservación, conciencia y cuidado de nuestro medio ambiente.
Zeballos (2005) estudio el impacto ecológico y ético de un proyecto de educación ambiental, desde el punto de vista teórico práctico, en un colegio donde habían desarrollado las áreas verdes en dicho centro, pero ubicado en una zona de naturaleza desértica, correspondiente a escolares que viven en el $\mathrm{Km} 38.5$ de la carretera Panamericana norte del distrito de Ventanilla, en la zona de Zapallal. Se halló un impacto positivo en las actitudes hacia el medio ambiente del proyecto de educación ambiental.

\section{MÉTODO}

La presente investigación es de nivel descriptivo de tipo sustantivo o básico, y de diseño descriptivo comparativo (Sánchez y Reyes, 2006). La población total estuvo integrada por 19235 estudiantes que asisten a la UGEL 08 Cañete, según el listado de la UGEL. De los cuales, 404 fueron estudiantes que asisten a escuelas rurales y 18831 que asisten a escuelas urbanas de la ciudad de Cañete.

El diseño en la selección de la muestra fue de tipo probabilístico de tipo estratificado, obteniéndose para dicha muestra un total de 743 estudiantes.

La variable de estudio fue Actitudes hacia la conservación del medio ambiente, la cual es definida conceptualmente como "la predisposición de un sujeto para aceptar o rechazar una determinada propuesta que supone la preservación de los recursos naturales, ambientales y su uso sostenible" (Yarlequé, 2004, p. 25).

La variable fue evaluada a través de la escala de Actitudes hacia la conservación del medio ambiente de Yarlequé (2004), la 
cual mide los aspectos cognitivo, reactivo

operacionalización de la variable).

y afectivo de dicha actitud (ver matriz de

\section{Matriz de operacionalización de la variable}

\begin{tabular}{|c|c|c|}
\hline Dimensiones & Indicadores & Ítems \\
\hline Cognitiva & $\begin{array}{l}\text { Es un conjunto de elementos informativos, de } \\
\text { experiencias que le permiten al individuo emitir } \\
\text { opiniones, juicios de valor y creencias acerca de su } \\
\text { entorno (Zimmermann, 2005). }\end{array}$ & $\begin{array}{l}1,3,4,5,6,8,9,12,13,21, \\
23,26,27,28,29,31 \text { у } 37\end{array}$ \\
\hline Reactiva & $\begin{array}{l}\text { Hace referencia a los sentimientos y emociones } \\
\text { que inspira a la persona en relación a su entorno, en } \\
\text { términos de atributos, los cuales pueden ser según } \\
\text { juicio de valor, es decir en todas las variantes de } \\
\text { agradables o desagradables, según sea el caso } \\
\text { (Zimmermann, 2005). }\end{array}$ & $\begin{array}{l}2,7,10,11,14,15,16,17, \\
18,19,22.30,33,34,35 \mathrm{y} \\
36\end{array}$ \\
\hline Afectiva & $\begin{array}{l}\text { Son disposiciones tanto positivas como negativas } \\
\text { sobre la relación con el ambiente físico y socio- } \\
\text { cultural, es decir si se comportan de acuerdo a las } \\
\text { reglas o normar previstas (Zimmermann, 2005). }\end{array}$ & $20,24,25$ у 32 \\
\hline
\end{tabular}

La escala es de tipo Likert en la cual los participantes debían escoger entre las siguientes alternativas: totalmente de acuerdo, de acuerdo, ni de acuerdo ni en desacuerdo, en desacuerdo o totalmente en desacuerdo. El instrumento estuvo integrado por 37 ítems, correspondiente a las dimensiones cognitiva, reactiva $\mathrm{y}$ afectiva.

Originalmente se obtuvo la confiabilidad bajo la modalidad test-retest con un grupo piloto de 52 sujetos. con 52 días de intervalo entre prueba y prueba en su aplicación (Yarlequé, 2004). A los puntajes obtenidos se les aplicó la fórmula producto momento de Pearson, arrojando un coeficiente general de correlación de 0,88 . En el componente cognitivo fue de 0,86 ; en el componente reactivo es de 0,92 y en el componente afectivo no se hizo la prueba pues técnicamente, afirmó el autor que no es posible por el escaso número de ítems.

En el desarrollo del presente estudio se aplicó el estadístico alfa de Cronbach, en lo que respecta a la evidencia de confiablidad, obteniendo un coeficiente de ,737 que demuestra su poder discriminativo de la prueba y por lo tanto confiable. En lo que respecta al análisis de ítems, se obtuvo una correlación ítems- test de ,19 y por encima de ella en todos los casos, salvo en los ítems 3, 4, 6, 13 y 20 que alcanza un coeficiente por debajo de ,19. Estos ítems no se anularon en la medida que no afectaban la unidad de la prueba.

Para los efectos del procesamiento estadístico de los datos se procedió 
al análisis descriptivo de la variable estudiada, luego análisis de la bondad de ajuste a la curva normal mediante la prueba de Kolmogorov-Smirnov y finalmente la inferencia estadística.

\section{Análisis descriptivo de la variable estudiada}

Tabla 1

Rangos totales en los componentes cognitivo, reactivo y afectivo

\begin{tabular}{clc}
\hline COMPONENTES & Escala & Porcentaje \\
\hline \multirow{2}{*}{ Cognitivo } & Bajo & $0.1 \%$ \\
& Medio & $65.3 \%$ \\
& Alto & $34.6 \%$ \\
& Total & $100 \%$ \\
Reactivo & Medio & $40.4 \%$ \\
& Alto & $59.6 \%$ \\
Afectivo & Total & $100 \%$ \\
& Bajo & $0.8 \%$ \\
& Medio & $34.7 \%$ \\
& Alto & $64.5 \%$ \\
& Total & $100 \%$ \\
\hline
\end{tabular}

En la tabla 1 se puede notar que en el componente cognitivo, del total de encuestados, el $0.1 \%$ tiene un nivel bajo, el $65.3 \%$ un nivel medio y el $34.6 \%$ un nivel alto. También se puede apreciar que, en el componente reactivo, del total de encuestados, el 40.4\% tiene un nivel medio $y$ el $59.6 \%$ un nivel alto. Finalmente, en el componente afectivo se aprecia que del total de encuestados, el $0.8 \%$ tiene un nivel bajo, el $35.5 \%$ un nivel medio y el $64.5 \%$ un nivel alto.

\section{Ajuste normal de la muestra}

\section{Tabla 2}

Resultado sobre el ajuste normal de la muestra a través del estadístico de Kolmorov - Smirnov

\begin{tabular}{lcc}
\hline Dimensiones & $\mathrm{Z}$ & Sig. \\
\hline Total escala &, 056 &, 000 \\
Nivel cognitivo &, 056 &, 000 \\
Nivel reactivo &, 051 &, 000 \\
Nivel afectivo &, 129 & .000 \\
\hline
\end{tabular}

n: $143 \mathrm{p}<, 005$ 
Se aplicó la prueba de normalidad de Kolmorov - Smirnov obteniéndose en todos los casos diferencias estadísticamente significativas, optando por la prueba no paramétrica para dar respuesta a los objetivos planteados.

\section{Tabla 3}

Comparación de los estudiantes del nivel secundario de escuelas rurales y urbanas de la UGEL 08

\begin{tabular}{lcccc}
\hline Dimensiones & $\begin{array}{c}\text { Urbano } \\
\text { R.M }\end{array}$ & $\begin{array}{c}\text { Rural } \\
\text { R.M }\end{array}$ & Z & Sig. \\
\hline Total escala & 362,55 & 388,39 & $-1,563$ &, 118 \\
Total cognitiva & 355,29 & 402,09 & $-2,832$ &, 005 \\
Total reactiva & 372,94 & 368,78 &,- 252 &, 801 \\
Total afectiva & 371,39 & 371,72 &,- 020 &, 984 \\
\hline
\end{tabular}

n: $763 \mathrm{p}<, 005$

En la tabla 3 se presenta la comparación de las actitudes hacia la conservación ambiental entre escolares de zona rurales y urbanas obteniendo lo siguiente:

Desde el punto de vista de la totalidad de la escala, se obtiene un z: $-1,563$ y p: ,118; observándose que no existen diferencias significativas entre ambos. Estos resultados se repiten en la dimensión reactiva $y$ afectiva, en la cual se obtiene un z: -,252 y p: ,801 en la dimensión reactiva y un $\mathrm{z}$ : -,020 y p: ,984 en la dimensión afectiva. Sin embargo, en la dimensión cognitiva, sí se observan diferencias estadísticamente significativas: $\mathrm{z}:-2,832 \mathrm{y}$ p:, 005 .

\section{Tabla 4}

Comparación de los estudiantes del nivel secundario varones y mujeres de zonas rurales de la UGEL 08

\begin{tabular}{lcccc}
\hline Dimensiones & $\begin{array}{c}\text { Femenino } \\
\text { R.M }\end{array}$ & $\begin{array}{c}\text { Varón } \\
\text { R.M }\end{array}$ & $Z$ & Zig. \\
\hline Total escala & 258,09 & 232,90 & $-2,004$ &, 045 \\
Total cognitiva & 247,57 & 240,13 &,- 570 &, 589 \\
Total reactiva & 249,78 & 232,47 & $-2,091$ &, 037 \\
Total afectiva & 265,88 & 228,64 & $-2,888$ &, 004 \\
\hline
\end{tabular}

n: $131 \mathrm{p}<, 005$ 
En la tabla 4 se presenta los resultados de la comparación de acuerdo al género entre estudiantes de zona rural, observándose lo siguiente:

Desde el punto de vista de la escala total se obtiene un z: -2,004 y p: ,045. observándose diferencias significativas, al igual que en la dimensión afectiva en la cual se obtiene un z: - 2,888 y $p$ :,004, así como en la dimensión reactiva, en la cual se obtiene un z: - 2, 091 y un $p$ : ,037. En todos los casos las puntuaciones van a favor de la mujer.

En la tabla 5 se presenta los resultados de la comparación de acuerdo con el género entre estudiantes varones y mujeres de zonas urbanas.

Tabla 5

Comparación de los estudiantes del nivel secundario varones y mujeres de zonas urbanas de la UGEL 08

\begin{tabular}{lcccc}
\hline Dimensiones & $\begin{array}{c}\text { Femenino } \\
\text { R.M }\end{array}$ & $\begin{array}{c}\text { Varón } \\
\text { R.M }\end{array}$ & Z & Sig. \\
\hline Total escala & 132,97 & 124,88 &,- 872 &, 383 \\
Total cognitiva & 127,75 & 130,30 &,- 275 &, 784 \\
Total reactiva & 136,04 & 121,68 & $-1,551$ &, 121 \\
Total afectiva & 135,36 & 122,38 & $-1,418$ &, 156 \\
\hline
\end{tabular}

n: $632 \mathrm{p}<, 005$

Desde el punto de vista de la escala total no se observa diferencia significativa entre estudiantes varones y mujeres de la zona urbana, se obtiene un z: -,872 y p: ,383. Dichos resultados se mantienen en las otras dimensiones, en el sentido de que no se observa diferencia significativa de la escala de actitudes de conservación del medio ambiente en las dimensiones cognitiva, reactiva y afectiva.

\section{Tabla 6}

Comparación de los estudiantes del nivel secundario varones de zona urbana y de zona rural de la UGEL 08

\begin{tabular}{lcccc}
\hline Dimensiones & $\begin{array}{c}\text { Varón urbano } \\
\text { R.M }\end{array}$ & $\begin{array}{c}\text { Varón rural } \\
\text { R.M }\end{array}$ & $\mathrm{Z}$ & Sig. \\
\hline Total escala & 157,03 & 163,02 &,- 572 &, 567 \\
Total cognitiva & 153,50 & 168,06 & $-1,391$ &, 164 \\
Total reactiva & 160,51 & 158,05 &,- 235 &, 814 \\
Total afectiva & 162,36 & 155,42 &,- 673 &, 501 \\
\hline
\end{tabular}

n: $422 \mathrm{p}<, 005$ 
En la tabla 6 se presenta los resultados de la comparación de los estudiantes varones de zona urbana y rural, en cuanto a las actitudes de la conservación del medio ambiente, en la cual se observa lo siguiente: Desde el punto de vista de la escala total se obtiene un $\mathrm{z}:-, 572$ y p: ,567, no observándose diferencias significativas. Dichos resultados se mantienen en función a las otras dimensiones, cognitiva, reactiva y afectiva.

\section{Tabla 7}

Comparación de los estudiantes del nivel secundario mujeres de zonas urbana y de zona rural de la UGEL 08

\begin{tabular}{lcccc}
\hline Dimensiones & $\begin{array}{c}\text { Mujer urbana } \\
\text { R.M }\end{array}$ & $\begin{array}{c}\text { Mujer rural } \\
\text { R.M }\end{array}$ & $Z$ & Z \\
\hline Total escala & 207,70 & 223,84 & $-1,240$ &, 215 \\
Total cognitiva & 202,99 & 235,00 & $-2,461$ &, 014 \\
Total reactiva & 214,31 & 208,21 &,- 469 &, 639 \\
Total afectiva & 212,18 & 213,25 &,- 084 &, 933 \\
\hline
\end{tabular}

n: $321 \mathrm{p}<, 005$

En la tabla 7 se presenta los resultados de la comparación de los estudiantes mujeres de zona urbana y rural, en cuanto a las actitudes de la conservación del medio ambiente, en la cual se obtiene lo siguiente:

Desde el punto de vista de la escala total se obtiene un $z:-1,240 y p:$,215., no observándose diferencias significativas. Dichos resultados se mantienen en función a las otras dimensiones, reactiva y afectiva, más no en la dimensión cognitiva, en la cual sí se observa diferencia significativa, observándose un $z$ : - 1, 240 y $p:, 014$.

\section{DISCUSIÓN}

Los resultados descritos constituyen la base para el análisis de las actitudes de conservación hacia el medio ambiente por parte del contexto escolar estudiado, además, sirven de reflexión sobre las posturas o confirmación de lo que viene sucediendo en las investigaciones con respecto al conocimiento, estados emocionales y reacciones en relación con el cuidado del medio ambiente.

Desde el punto de vista global se aprecia que no existen diferencias significativas entre los estudiantes de zona urbano y de zona rural, que llevaría a pensar que la visión sobre la naturaleza y su conservación es igual entre los estudiantes de ambas zonas, sin embargo, al analizar las dimensiones de la escala de actitudes se puede observar diferencia en la dimensión cognitiva, cuyos puntajes van a favor de los estudiantes de zona rural, lo que lleva a describir un comportamiento actitudinal más firme y comprometido en dichos estudiantes. Probablemente la cercanía al 
contexto rural influye en su vida y en su comportamiento hacia la conservación del medio ambiente. Al respecto, los estudios de Yarlequé (2004) refieren que el nivel de instrucción no determina las diferencias, o en todo caso no es muy claro en este aspecto.

Al comparar las actitudes hacia la conservación del medio ambiente en estudiantes varones y mujeres de zona rural, se puede observar diferencias notables a favor de las estudiantes mujeres, en los puntajes totales y en las dimensiones reactivas y afectivas. Esto a pesar que ambos, varones y mujeres, vivan en un ambiente rural, sin embargo, en las mujeres se aprecia una mayor sensibilidad en la problemática medio ambiental, así como una actitud más comprometida hacia el cuidado del medio ambiente. Por ello, se visualiza un gran liderazgo, interés y madurez de las mujeres en el campo. Kals (2009) manifiesta que hay una relación positiva en la afinidad emocional hacia la naturaleza en adolescentes y la voluntad de proteger el medio ambiente, el cual se visualiza en un mayor compromiso con el medio ambiente.

En lo que respecta a los estudiantes varones y mujeres de zona urbana, no se observó diferencia alguna. Esto puede ser traducido en que los estudiantes, tanto varones y mujeres, de zona urbana no viven cerca del campo y no dependen aparentemente de dicho contexto para sobrevivir, pero también se acentúa la experiencia de vivir en la ciudad, el cual es considerado como un ambiente artificial creado por el hombre, con sus cualidades y sus defectos. Sin embargo, en la comparación de género en zona rural, la diferencia es más clara a favor de la mujer en función a los varones y mujeres de zona urbana. De alguna forma resalta la imagen de la mujer de la zona del campo y su probable liderazgo en este aspecto. Sin embargo, a pesar de la presencia importante de la mujer del campo en este aspecto, Pereyra (2008) indica que el género no guarda relación con el respeto hacia el medio ambiente y al desarrollo sostenible.

En relación a la comparación entre estudiantes varones de zona urbana $\mathrm{y}$ de zona rural, se observa claramente que no hay diferencias estadísticamente significativas en las actitudes hacia la conservación del medio ambiente, es decir, que las actitudes hacia la conservación ambiental se encuentran en un mismo nivel, pero este comportamiento hacia el medio ambiente es bajo. Lo que sí es notable en los resultados, es el aspecto afectivo de las mujeres que viven en zonas rurales. Sin embargo, los estudios de Villacorta et al. (2008) revelan resultados distintos a lo alcanzado en el presente estudio, en la medida que no observa diferencias en las actitudes hacia la conservación del medio ambiente en estudiantes de zona urbana y rural; claro que tal estudio fue realizado en la región de Loreto que es una región muy cercana a la Amazonía, razón por la cual no debería sorprender dicho resultado.

En lo que respecta a las actitudes hacia la conservación del medio ambiente en estudiantes mujeres de zona urbana y de zona rural, se observan diferencias en el aspecto cognitivo a favor de las estudiantes mujeres de la zona rural. Hay que resaltar la sensibilidad de las estudiantes mujeres de ambas zonas, tanto urbana como rural, que en ambos casos no se diferencia, sin embargo, en la dimensión cognitiva sí existe diferencia. Esto es obvio en la 
medida de la cercanía de las estudiantes mujeres de zona rural a la naturaleza, que en realidad constituye su medio de vida.

Por lo demás, Wray-Lake (2010) indica que en los últimos años las actitudes favorables hacia el medio ambiente han disminuido considerablemente de manera general, el cual asume un riesgo para la sobrevivencia universal; por el contrario, González y Amérigo (1999) señalan que en la medida que las personas tienen actitudes proambientalistas se relacionan de forma positiva con el ecocentrismo. Es decir, las personas que tienen buenas actitudes hacia el medio ambiente son las que mejor cuidan nuestro planeta y, tanto la educación como la familia son el contexto básico de formación del comportamiento saludable hacia la naturaleza.

\section{CONCLUSIONES}

- Se aprecia diferencia en la dimensión cognitiva entre estudiantes de zona urbana y de zona rural, a favor de los estudiantes de zona rural.
- Se observa un mayor compromiso en las actitudes de conservación del medio ambiente en las estudiantes mujeres de zona rural que en los varones de dicha zona.

- En lo que respecta a los estudiantes varones y mujeres de zona urbana, no se observó diferencia alguna.

- No hay diferencias significativas en las actitudes hacia la conservación del medio ambiente, entre estudiantes varones de zona urbana y varones de zona rural.

- Hay diferencia significativa solo en la dimensión cognitiva entre estudiantes mujeres de zona rural y mujeres de la zona urbana. Las características personales de las estudiantes mujeres de zona rural son más favorables hacia la conservación del medio ambiente en cuanto al aspecto cognitivo, reactivo y afectivo. 


\section{REFERENCIAS}

Chapilliquen, M. (2007). Efectos del programa experimental Salvemos la Tierra en la formación de la conciencia ecológica: investigación realizada en alumnos del primer grado de educación secundaria de la I.E. No 1057 José Baquíjano y Carrillo del distrito de Lince (Tesis de maestría). Universidad Femenina del Sagrado Corazón, Lima, Perú.

Edquen e Ybañes (2013). Influencia del programa educativo sobre contaminación por residuos sólidos en el aprendizaje de los estudiantes del sexto grado I.E. Monseñor Juan Tomis Stack (Tesis de licenciatura). Universidad Católica Santo Toribio de Mogrovejo, Chiclayo, Perú.

Galli, F., Bedim, L., Bolzan de Campos, C. y Castellá Sarriera, J. (2013). Actitudes hacia el medio ambiente en la infancia. Revista Latinoamericana de Psicología, 45(3), 459-471. Recuperado de https://dialnet.unirioja.es/metricas/documentos/ARTREV/4665340

González, A. y Amérigo, M. (1999). Actitudes hacia el medio ambiente y conducta ecológica. Psichotema, 11(1), 13-25 Recuperado de https://www.unioviedo.es/reunido/ index.php/PST/article/view/7554/7418.

Holguín, H. (11 de diciembre del 2014). COP 20 y Lima, la sede que no es ejemplo. El Comercio. Recuperado de http://elcomercio.pe/opinion/columnistas/cop-20-y-sedeque-no-ejemplo-herbert-holguin-noticia-1777631?ref=portada_home.

Kals, E. (2009). Afinidad emocional de los adolescentes con la naturaleza: un estudio inter-societario. Recuperado de https://www.researchgate.net/publication/41845344 Adolescents'_Emotional_Affinity_toward_Nature_A_Cross-Societal_Study

Ministerio de Educación, MINEDU. (2019). Currículo Nacional: orientaciones para las reuniones con madres y padres de familia. Recuperado de http://www.minedu.gob.pe/ curriculo/madres-padres.php

Organización de las Naciones Unidas (2005). Objetivos de desarrollo del milenio informe de 2005. 30-35.

Pereyra, J. (2008). Estudio de la responsabilidad ciudadana y el medio ambiente en la ciudad de Lima (Tesis doctoral). Universidad Pontificia Católica del Perú. Lima.

Programa de las Naciones Unidas para el Desarrollo (2011). Informe sobre desarrollo humano 2011 sostenibilidad y equidad: un mejor futuro para todos. Recuperado de https://www1.undp.org/content/undp/es/home/librarypage/hdr/human developmentreport2011.html

Sánchez, H. y Reyes, C. (2006). Metodología y diseños en la investigación científica: aplicadas a la psicología, educación y ciencias sociales. Ed. Universitaria.

Villacorta, J., Villacorta, E., Vásquez, M., Reátegui, G., Ruiz, A., Vásquez, W. ... Flores, A. (2008). Actitudes hacia la conservación del medio ambiente de padres de familia, docentes y estudiantes de la zona urbana y rural de Belén. Recuperado de http://www. unapiquitos.edu.pe/oficinas/investigacion/descargas/2008/ARTICULO-JUDITH\%20 VILLACORTA.pdf 
Wray-Lake, L., Flanagan, C. A. \& Osgood, D. W. (2010). Examining trends in adolescent environmental attitudes, beliefs, and behaviors across three decades. Environ Behav. 42(1), 61-85. Recuperado de https://www.ncbi.nlm.nih.gov/pmc/articles/ PMC2790169/

Yarlequé, L. (2004). Actitudes hacia la Conservación Ambiental en Estudiantes de Educación Secundaria (Tesis doctoral). Universidad Nacional Mayor de San Marcos. Lima, Perú.

Zeballos, M. (2005). Impacto de un proyecto de educación ambiental en estudiantes de un colegio en una zona marginal de Lima (Tesis de maestría). Pontificia Universidad Católica del Perú. Lima, Perú.

Zinmermann, M. (2005). Ecopedagogía: el planeta en emergencia. Bogotá, DC, Colombia: ECOE

Fecha de recepción: 15 de abril de 2020

Fecha de aceptación: 12 de mayo de 2020 